\title{
One-Pot Synthesis of 5,6-Dihydro-4H-1,2-Oxazines by Cyclization of Ketoximes with Derivatives of Allylbenzene
}

\author{
Raad Kasim Yahya ${ }^{a^{*}}$, K.M.Lokanatha Rai ${ }^{a}$, Ebraheem Abdu Musad ${ }^{a}$ and P. T. Sowmya ${ }^{a}$ \\ $\mathrm{a}^{*}$ Department of Studies in Chemistry, University of Mysore, Mysore, 570006, India. \\ 1st author's E-mail: raadchem@gmail.com \\ 2nd E-mail: kmlrai@yahoo.com \\ 3rd E-mail: alhashmi.ibrahim@yahoo.com \\ $4^{\text {th }}$ E-mail: ptsowmya8@gmail.com
}

\section{ABSTRACT}

A new series of 5,6-dihydro-4H-1,2-oxazines were synthesized via hetero Diels-Alder reaction of $\alpha$-nitrosolefins with derivatives of allylbenzene. a-Nitrosoolefins were generated from ketoximes by the action of chloramine-T and triethylamine.

\section{Indexing terms/Keywords}

1,2-Oxazine, a-Nitrosoolefins, allylbenzene, Ketoximes, Cycloaddition

\section{Council for Innovative Research}

Peer Review Research Publishing System

Journal: Journal of Advances in Chemistry

Vol. 6, No. 1

editor@cirworld.com

www.cirworld.com, member.cirworld.com 


\section{INTRODUCTION}

[4+2] cycloaddition of a-nitrosoolefins with olefinic compounds yields 5,6-dihydro-4H-1,2-oxazines which are useful intermediates for the synthesis of bifunctional compounds. The nitrosoolefins which are used in the reaction are transient species [1-5]. 5,6-Dihydro-4H-1,2-oxazines which are of biological importance, viz. used as intermediates during the synthesis of glycosidase inhibitor analogues [6-8] and of functionalized pyrroles [9].Tetrahydro-4H-1,2-oxazines are the important structural constituents of many fungicides, herbicides [10] and microbicides [11]. Oxazines are used as key building blocks in the synthesis of natural products [12]. For instance, oxazine are used as synthones to synthesize pyrroles [13,14], pyrrolidine [15], pyridines [16] and $\gamma$ - lactones [17]. $\alpha$-Nitrosoolefins are usually generated in situ from $\alpha$ haloketoximes, by reaction with a heterogeneous base such as sodium carbonate in an organic solvent (dichloromethane, diethyl ether or methyl $t$-butyl ether) at room temperature [18-20]. 1,2-Oxazines are also available from alkenyl ketoximes by reacting them with $\mathrm{N}$-bromosuccinimide [21], trifluoroacetic acid [22] and UV mediated cyclizations of alkenylsubstituted oximes [23]. 1,2-Oxazines have also been prepared by base-mediated cyclizations of $\gamma$-chloroximes [24] and $\gamma$ sulfonyloximes [25]. Other synthetic approaches to 1,2-oxazines rely on Lewis-acid catalyzed reactions of allenoximes [26], acid-catalyzed cyclization of cyclopropyloximes [27] and on cyclizations of $y$-nitroketones [28]. Langer et al reported the synthesis of 1,2-oxazines by cyclization of oxime dianions with epibromohydrin [29]. Recently Goanker and Rai prepared 5,6-dihydro-4H-1,2-oxazines by generating a-nitrosolefins from ketoximes and further reacting them with olefinic compounds [30]. With this background, herein we report the cycloaddition reaction of different $\alpha$-nitrosolefins with various derivatives of allylbenzene.

\section{EXPERIMENTAL}

\section{Reagents and apparatus}

All chemicals used are lab grade chemicals and were used without further purification. All reactions were monitored by TLC, which was performed on Merk Alumina foil $60 \mathrm{~A}^{\circ}$ TLC plates with UV indicator. The solvent system used for developing the TLC plates was $\mathrm{CHCl}_{3}$ : petroleum ether (1:3). The ${ }^{1} \mathrm{H}$ NMR and ${ }^{13} \mathrm{C}$ NMR spectrum were measured on Jeol, using TMS as internal standard. The IR spectra (in KBr pellets) were recorded on JASCO FT/IR- 460/113257 spectrometer (Japan) in the wave number range of $4000-400 \mathrm{~cm}^{-1}$. The melting points were determined on Thomas Hoover. Mass spectra obtained on a Finnigan 4021. Elemental analyses were obtained on a Vario- EL instrument.

\section{General procedure for the synthesis of oxazine derivatives 5(a-r)}

A mixture of ketoxime $(5 \mathrm{mmol})$ and chloramine-T trihydrate $(5.1 \mathrm{mmol})$ in ethanol $(20 \mathrm{ml})$ was refluxed for 4 hours. The mixture was cooled to room temperature, triethylamine $(1 \mathrm{ml})$ was added and stirred at room temperature for $1 \mathrm{~h}$. After this, solution of allylbenzene $(5.1 \mathrm{mmol})$ in ethanol $(10 \mathrm{ml})$ was added and refluxed for $12-20$ hours . It was then concentrated under reduced pressure and the residue was extracted with ether $(50 \mathrm{ml})$. This extract was then washed with water (15 $\mathrm{ml})$, aqueous $5 \% \mathrm{NaOH}(30 \mathrm{ml})$ and dried over anhydrous sodium sulfate. The solvent was evaporated and the remaining solid was recrystallized from $10 \%$ ethanol in $n$-hexane to afford the pure compound.

\section{Synthesis of 6- benzyl-3-phenyl-5,6-dihydro-4H-1,2-oxazines (5a)}

Obtained from 1a $(0.66 \mathrm{~g}, 5 \mathrm{mmol})$, chloramine-T.3 $\mathrm{H}_{2} \mathrm{O}(1.43 \mathrm{~g}, 5.1 \mathrm{mmol})$ and $4 \mathrm{a}(1.43 \mathrm{~g}, 5.1 \mathrm{mmol})$ as a brown oil, Yield $65 \%$; mp 131-132 ${ }^{\circ} \mathrm{C}$; IR $\left(\mathrm{KBr}, \mathrm{cm}^{-1}\right): 1600(\mathrm{C}=\mathrm{C}), 1645(\mathrm{C}=\mathrm{N}) .{ }^{1} \mathrm{H}-\mathrm{NMR}\left(300 \mathrm{MHz}, \mathrm{CDCl}_{3}, \delta / \mathrm{ppm}\right): 1.82-2.04(\mathrm{~m}, 2 \mathrm{H}$, $\left.\mathrm{OCHCH}_{2}\right), 2.60-2.74\left(\mathrm{t}, J=7.2 \mathrm{~Hz}, 2 \mathrm{H}, \mathrm{CCH}_{2}\right), 2.78-2.87\left(\mathrm{~m}, 2 \mathrm{H}, \mathrm{Ph}-\mathrm{CH}_{2}\right), 3.75-3.82(\mathrm{~m}, 1 \mathrm{H}, \mathrm{OCH}), 7.20-7.73(\mathrm{~m}, 10 \mathrm{H}, \mathrm{Ar}-$ $\mathrm{H}),{ }^{13} \mathrm{C}$ NMR $\left(300 \mathrm{MHz}, \mathrm{CDCl}_{3}\right) ; 17.2,24.2,34.9,86.90,118.8,123.3,125.3,131.2,135.9,138.4,141.8,146.7,165.30$. Anal. Calcd. for $\mathrm{C}_{17} \mathrm{H}_{17} \mathrm{NO}$ (251.13): C, 81.24; H, 6.82; N, 5.57. Found: C, 81.44; H, 6.64; N, 5.33. LC-MS: m/z 252.26[M]

\section{6-(3,4-dimethoxybennzyl)-3-phenyl-5,6-dihydro-4H-1,2-oxazines (5b)}

Obtained from $1 \mathrm{a}(0.66 \mathrm{~g}, 5 \mathrm{mmol})$, chloramine-T. $3 \mathrm{H}_{2} \mathrm{O}(1.43 \mathrm{~g}, 5.1 \mathrm{mmol})$ and $\mathbf{4 b}(0.9 \mathrm{~g}, 5.1 \mathrm{mmol})$ as a yellow oil, Yield 52\%; mp 110-112 ${ }^{\circ} \mathrm{C}$; IR $\left(\mathrm{KBr}, \mathrm{cm}^{-1}\right): 1600(\mathrm{C}=\mathrm{C}), 1644(\mathrm{C}=\mathrm{N}) .{ }^{1} \mathrm{H}-\mathrm{NMR}\left(300 \mathrm{MHz}, \mathrm{CDCl}_{3}, \delta / \mathrm{ppm}\right): 1.83-2.02(\mathrm{~m}, 2 \mathrm{H}$, $\left.\mathrm{OCHCH}_{2}\right), 2.65-2.76\left(\mathrm{t}, J=7.2 \mathrm{~Hz}, 2 \mathrm{H}, \mathrm{CCH}_{2}\right), 2.79-2.86\left(\mathrm{~m}, 2 \mathrm{H}, \mathrm{Ph}-\mathrm{CH}_{2}\right), 3.72-3.81(\mathrm{~m}, 1 \mathrm{H}, \mathrm{OCH}), 3.86-3.92(\mathrm{~m}, 6 \mathrm{H}$, $\left.\mathrm{OCH}_{3}\right)$, ) , 6.70-6.79 (m, 3H, Ar-H), 7.24-7.72 (m, 5H, Ar-CH), ). $\left.{ }^{13} \mathrm{C} \mathrm{NMR} \mathrm{(300} \mathrm{MHz,} \mathrm{CDCl}_{3}\right) ; 15.6,25.6,40.6,59.9,85.8$, 109.8, 115.4, 119.7, 128.2, 130.3, 134.3, 136.4, 139.7, 146.3, 150.5, 166.3. Anal. Calcd. for $\mathrm{C}_{19} \mathrm{H}_{21} \mathrm{NO}_{3}(311.15): \mathrm{C}$ 73.29; H, 6.80; N, 4.50. Found: C, 73.50; H, 6.54; N, 4.60. LC-MS: m/z $312.25[M]^{+}$.

\section{2-methoxy-4-(3-phenyl-5,6-dihydro-4H-1,2-oxazin-6-yl) methyl\} phenol (5c)}

Obtained from $1 \mathrm{a}(0.66 \mathrm{~g}, 5 \mathrm{mmol})$, chloramine-T.3 $\mathrm{H}_{2} \mathrm{O}(1.43 \mathrm{~g}, 5.1 \mathrm{mmol})$ and $4 \mathrm{c}(0.85 \mathrm{~g}, 5.1 \mathrm{mmol})$ as a white solid, Yield 62\%; mp 87-88 ${ }^{\circ} \mathrm{C}$; IR ( $\left.\mathrm{KBr}, \mathrm{cm}^{-1}\right): 1600(\mathrm{C}=\mathrm{C}), 1646(\mathrm{C}=\mathrm{N}), 3350(\mathrm{HO}) .{ }^{1} \mathrm{H}-\mathrm{NMR}\left(300 \mathrm{MHz}, \mathrm{CDCl}_{3}, \delta / \mathrm{ppm}\right): 1.85-2.03(\mathrm{~m}$, $\left.2 \mathrm{H}, \mathrm{OCHCH}_{2}\right), 2.64-2.75\left(\mathrm{t}, J=7.2 \mathrm{~Hz}, 2 \mathrm{H}, \mathrm{CCH}_{2}\right), 2.79-2.84\left(\mathrm{~m}, 2 \mathrm{H}, \mathrm{PhCH}_{2}\right), 3.74-3.81(\mathrm{~m}, 1 \mathrm{H}, \mathrm{OCH}), 3.86(\mathrm{~s}, 3 \mathrm{H}$, $\left.\mathrm{OCH}_{3}\right), 5.65(\mathrm{~s}, 1 \mathrm{H}, \mathrm{OH}), 6.50-6.62(\mathrm{~m}, 3 \mathrm{H}, \mathrm{Ar}-\mathrm{H}), 7.50(\mathrm{t}, \mathrm{J}=6 \mathrm{~Hz}, 2 \mathrm{H}, \mathrm{Ar}-\mathrm{H}), 7.70-7.80(\mathrm{~m}, 3 \mathrm{H}, \mathrm{Ar}-\mathrm{H}) .{ }^{13} \mathrm{C} \mathrm{NMR}(300 \mathrm{MHz}$, $\left.\mathrm{CDCl}_{3}\right)$; 16.7, 23.2, 42.2, 60.5, 85.9, 110.1, 118.5, 121.9, 125.5, 128.8, 132.2, 133.4, 135.5, 145.8, 148.5, 166.7. Anal. Calcd. for $\mathrm{C}_{18} \mathrm{H}_{19} \mathrm{NO}_{3}$ (297.14): C, 72.71; H, 6.44; N, 4.71. Found: C, 72.50; H, 6.70; N, 4.60. LC-MS: m/z 298.26 [M] .

\section{6-benzyl-3-p- tolyl-5,6- dihydro-4H-1,2-oxazine (5d)}

Obtained from $\mathbf{1 b}(0.74 \mathrm{~g}, 5 \mathrm{mmol})$, chloramine-T. $3 \mathrm{H}_{2} \mathrm{O}(1.43 \mathrm{~g}, 5.1 \mathrm{mmol})$ and $4 \mathrm{a}(0.6 \mathrm{~g}, 5.1 \mathrm{mmol})$ as a white solid, Yield $68 \%$; mp 124-126 ${ }^{\circ} \mathrm{C}$; IR $\left(\mathrm{KBr}, \mathrm{cm}^{-1}\right): 1600(\mathrm{C}=\mathrm{C}), 1644(\mathrm{C}=\mathrm{N}), 2995\left(\mathrm{CH}\right.$-aliph.). ${ }^{1} \mathrm{H}-\mathrm{NMR}\left(300 \mathrm{MHz}, \mathrm{CDCl}_{3}, \delta / \mathrm{ppm}\right): 1.86-$ 
$2.05\left(\mathrm{~m}, 2 \mathrm{H}, \mathrm{OCHCH}_{2}\right), 2.39\left(\mathrm{~s}, 3 \mathrm{H}, \mathrm{CH}_{3}\right), 2.64-2.76\left(\mathrm{t}, \mathrm{J}=7.2 \mathrm{~Hz}, 2 \mathrm{H}, \mathrm{CCH}_{2}\right), 2.79-2.88\left(\mathrm{~m}, 2 \mathrm{H}, \mathrm{PhCH}_{2}\right), 3.76-3.84(\mathrm{~m}$, $1 \mathrm{H}, \mathrm{OCH}), 7.10$ (dd, $J=6 \mathrm{~Hz}, J=2 \mathrm{~Hz}, 2 \mathrm{H}, \mathrm{Ar}-\mathrm{H}), 7.20$ (t, $J=6 \mathrm{~Hz}, 2 \mathrm{H}, \mathrm{Ar}-\mathrm{H}), 7.35(\mathrm{~m}, 3 \mathrm{H}, \operatorname{Ar}-\mathrm{H}), 7.58(\mathrm{dd}, J=6 \mathrm{~Hz}, J=$ $2 \mathrm{~Hz}, 2 \mathrm{H}, \mathrm{Ar}-\mathrm{H}) .{ }^{13} \mathrm{C} N M R\left(300 \mathrm{MHz}, \mathrm{CDCl}_{3}\right) ; 15.5,23.9,25.7,41.4,87.6,122.9,126.9,129,7,131.3,133.4,135.2,139.9$, 170. Anal. Calcd. for $\mathrm{C}_{18} \mathrm{H}_{19} \mathrm{NO}$ (265.35): C, 81.47; H ,7.22; N, 5.28. Found: C,81.66; H, 6.99; N, 6.66. LC-MS: m/z 266.44 $[\mathrm{M}]^{+}$.

\section{6-(3,4-dimethoxybenzyl)-3-p-tolyl-5,6-dihydro-4H-oxazine (5e)}

Obtained from $\mathbf{1 b}(0.74 \mathrm{~g}, 5 \mathrm{mmol})$, chloramine-T. $3 \mathrm{H}_{2} \mathrm{O}(1.43 \mathrm{~g}, 5.1 \mathrm{mmol})$ and $\mathbf{4 b}(0.9 \mathrm{~g}, 5.1 \mathrm{mmol})$ as a colorless solid, Yield 62\%; mp110-112 ${ }^{\circ} \mathrm{C}$; IR $\left(\mathrm{KBr}, \mathrm{cm}^{-1}\right): 1598(\mathrm{C}=\mathrm{C}), 1647(\mathrm{C}=\mathrm{N}), 2994\left(\mathrm{CH}\right.$-aliph.). ${ }^{1} \mathrm{H}-\mathrm{NMR}(300 \mathrm{MHz}, \mathrm{CDCl}, \delta / \mathrm{ppm}):$ 1.84-2.03 (m, 2H, OCHCH 2$), 2.40\left(\mathrm{~s}, 3 \mathrm{H}, \mathrm{CH}_{3}\right), 2.66-2.76\left(\mathrm{t}, J=7.2 \mathrm{~Hz}, 2 \mathrm{H}, \mathrm{CCH}_{2}\right), 2.79-2.88\left(\mathrm{~m}, 2 \mathrm{H}, \mathrm{PhCH}_{2}\right), 3.77-3.82$ $(\mathrm{m}, 1 \mathrm{H}, \mathrm{OCH}), 3.87-3.93\left(\mathrm{~m}, 6 \mathrm{H}, \mathrm{OCH}_{3}\right), 6.55-6.66(\mathrm{~m}, 3 \mathrm{H}, \mathrm{Ar}-\mathrm{H}), 7.20(\mathrm{dd}, \mathrm{J}=6 \mathrm{~Hz}, \mathrm{~J}=2 \mathrm{~Hz}, 2 \mathrm{H}, \mathrm{Ar}-\mathrm{H}), 7.65(\mathrm{dd}, J=6$ $\mathrm{Hz}, J=2 \mathrm{~Hz}, 2 \mathrm{H}, \mathrm{Ar}-\mathrm{H}) .{ }^{13} \mathrm{C}$ NMR $\left(300 \mathrm{MHz} \mathrm{CDCl}_{3}\right) ; 15.6,23.9,24.8,40.8,59.7,88.1,115.6,117.8,126.7,129.7,132.4$, 136.4, 137.8, 146.6, 148.9, 155.8, 166.5. Anal. Calcd. for $\mathrm{C}_{20} \mathrm{H}_{23} \mathrm{NO}_{3}$ (325.17): C, 73.82; $\mathrm{H}, 7.12 ; \mathrm{N}, 4.30$. Found: C,73.99; H, 6.89; N, 4.50. LC-MS: m/z $326.29[\mathrm{M}]^{+}$.

\section{2-methoxy-4-[(3-p-tolyl-5,6-dihydro-4H-1,2-oxazin-6-yl)-methyl]-phenol (5f)}

Obtained from 1b $(0.74 \mathrm{~g}, 5 \mathrm{mmol})$, chloramine- $\mathrm{T}^{3} \mathrm{H}_{2} \mathrm{O}(1.43 \mathrm{~g}, 5.1 \mathrm{mmol})$ and $4 \mathrm{c}(0.85 \mathrm{~g}, 5.1 \mathrm{mmol})$ as a pale yellow solid, Yield 58\%; mp 63-64 ${ }^{\circ} \mathrm{C}$; IR $\left(\mathrm{KBr}, \mathrm{cm}^{-1}\right)$ : $1600(\mathrm{C}=\mathrm{C}), 1644(\mathrm{C}=\mathrm{N}), 2994\left(\mathrm{CH}\right.$-aliph.), $3450(\mathrm{HO}) .{ }^{1} \mathrm{H}-\mathrm{NMR}(300 \mathrm{MHz}$, $\left.\mathrm{CDCl}_{3} \delta / \mathrm{ppm}\right): 1.84-2.04\left(\mathrm{~m}, 2 \mathrm{H}, \mathrm{OCHCH}_{2}\right), 2.38\left(\mathrm{~s}, 3 \mathrm{H}, \mathrm{CH}_{3}\right), 2.63-2.73\left(\mathrm{t}, J=7.2 \mathrm{~Hz}, 2 \mathrm{H}, \mathrm{CCH}_{2}\right), 2.77-2.85(\mathrm{~m}, 2 \mathrm{H}$, $\left.\mathrm{PhCH}_{2}\right), 3.77-3.83(\mathrm{~m}, 1 \mathrm{H}, \mathrm{OCH}), 3.88\left(\mathrm{~s}, 3 \mathrm{H}, \mathrm{OCH}_{3}\right), 5.42(\mathrm{~s}, 1 \mathrm{H}, \mathrm{OH}), 6.55-6.78(\mathrm{~m}, 3 \mathrm{H}, \mathrm{Ar}-\mathrm{H}), 7.15(\mathrm{dd}, J=6 \mathrm{~Hz}, J$ $=2 \mathrm{~Hz}, 2 \mathrm{H}, \mathrm{Ar}-\mathrm{H}), 7.70(\mathrm{dd}, J=6 \mathrm{~Hz}, J=2 \mathrm{~Hz}, 2 \mathrm{H}, \mathrm{Ar}-\mathrm{H}) .{ }^{13} \mathrm{C} \mathrm{NMR}\left(300 \mathrm{MHz}, \mathrm{CDCl}_{3}\right) ; 18.6,24.9,26.9,40.4,55.7,86.9$, 110.9, 117.6, 120.8, 125.6, 129.1, 131.6, 137.7, 142.1, 147.5, 149.6, 164.3. Anal. Calcd. for $\mathrm{C}_{19} \mathrm{H}_{21} \mathrm{NO}_{3}(315.15)$ : $\mathrm{C}$, 73.29; H, 6.80; N, 4.50.: Found C, 73.20; H, 6.90; N, 4.80. LC-MS: m/z $316.28[\mathrm{M}]^{+}$.

\section{6-benzyl-3-(4-chlorophenyl)-5,6-dihydro-4H-1,2-oxazine $(5 \mathrm{~g})$}

Obtained from 1c $(0.85 \mathrm{~g}, 5 \mathrm{mmol})$, chloramine-T. $3 \mathrm{H}_{2} \mathrm{O}(1.43 \mathrm{~g}, 5.1 \mathrm{mmol})$ and $4 \mathbf{a}(0.6 \mathrm{~g}, 5.1 \mathrm{mmol})$ as a colorless solid, Yield 60\%; mp74-76 ${ }^{\circ} \mathrm{C}$; IR $\left(\mathrm{KBr}, \mathrm{cm}^{-1}\right): 1598(\mathrm{C}=\mathrm{C}), 1646(\mathrm{C}=\mathrm{N}), 2994\left(\mathrm{CH}\right.$-aliph.). ${ }^{1} \mathrm{H}-\mathrm{NMR}\left(300 \mathrm{MHz}, \mathrm{CDCl}_{3}, \delta / \mathrm{ppm}\right)$ : 1.87-2.03 (m, 2H, OCHCH 2$), 2.64-2.74\left(\mathrm{t}, J=7.2,2 \mathrm{H}, \mathrm{CCH}_{2}\right), 2.77-2.87\left(\mathrm{~m}, 2 \mathrm{H}, \mathrm{PhCH}_{2}\right), 3.74-3.82(\mathrm{~m}, 1 \mathrm{H}, \mathrm{OCH}), 7.20(\mathrm{t}$, $J=6 \mathrm{~Hz}, 2 \mathrm{H}, \mathrm{Ar}-\mathrm{H}), 7.35(\mathrm{~m}, 3 \mathrm{H}, \mathrm{Ar}-\mathrm{H}), 7.50(\mathrm{~d}, \mathrm{~J}=6 \mathrm{~Hz}, 2 \mathrm{H}, \mathrm{ArH}), 7.65(\mathrm{~d}, J=6 \mathrm{~Hz}, 2 \mathrm{H}, \operatorname{Ar}-\mathrm{H}) .{ }^{13} \mathrm{C} \mathrm{NMR}(300 \mathrm{MHz}$, $\left.\mathrm{CDCl}_{3}\right) ; 16.7,23.2,40.3,86.6,122.4,128.1,130.1,133.2,136.5,140.9,165.8$. Anal. Calcd. for $\mathrm{C}_{17} \mathrm{H}_{16} \mathrm{CINO}(285.09): \mathrm{C}_{\text {, }}$ 71.45; H, 5.64; N, 4.90. Found: C, 71.50; H, 5.34; N, 5,08. LC-MS: m/z $285\left[\mathrm{M}^{+}, 287.18\left[\mathrm{M}^{+}+2\right]\right.$,

\section{3-(4-chlorophenyl)-6-(3,4-dimethoxybenzyl)-5,6-dihydro-4H-1,2-oxazine (5h)}

Obtained from $1 \mathrm{c}(0.85 \mathrm{~g}, 5 \mathrm{mmol})$, chloramine-T. $3 \mathrm{H}_{2} \mathrm{O}(1.43 \mathrm{~g}, 5.1 \mathrm{mmol})$ and $4 \mathbf{b}(0.9 \mathrm{~g}, 5.1 \mathrm{mmol})$ as a pale yellow solid, Yield 69\%; mp 67-68 ${ }^{\circ} \mathrm{C}$; IR $\left(\mathrm{KBr}, \mathrm{cm}^{-1}\right): 1600(\mathrm{C}=\mathrm{C}), 1647(\mathrm{C}=\mathrm{N}), 2990$ (CH-aliph.). ${ }^{1} \mathrm{H}-\mathrm{NMR}\left(300 \mathrm{MHz}, \mathrm{CDCl}_{3}, \delta / \mathrm{ppm}\right)$ : 1.84-2.01 (m, $\left.2 \mathrm{H}, \mathrm{OCHCH}_{2}\right), 2.65-2.74\left(\mathrm{t}, J=7.2 \mathrm{~Hz}, 2 \mathrm{H}, \mathrm{CCH}_{2}\right), 2.78-2.87\left(\mathrm{~m}, 2 \mathrm{H}, \mathrm{PhCH}_{2}\right), 3.73-3.83(\mathrm{~m}, 1 \mathrm{H}, \mathrm{OCH}), 3.91-$ $3.96\left(\mathrm{~m}, 6 \mathrm{H}, \mathrm{OCH}_{3}\right), 6.55-6.66(\mathrm{~m}, 3 \mathrm{H}, \mathrm{Ar}-\mathrm{H}), 7.40(\mathrm{~d}, \mathrm{~J}=6 \mathrm{~Hz}, 2 \mathrm{H}, \mathrm{Ar}-\mathrm{H}), 7.60(\mathrm{~d}, \mathrm{~J}=6 \mathrm{~Hz}, 2 \mathrm{H}, \operatorname{Ar}-\mathrm{H}) .{ }^{3} \mathrm{C} \mathrm{NMR}(300 \mathrm{MHz}$, $\left.\mathrm{CDCl}_{3}\right) ; 15.9,25.5,41.3,57.3,85.4,109.9,117.9,120.8,128.4,130.2,133.2,135.7,139.9,148.4,150.5$, 165.1. Anal. Calcd. for $\mathrm{C}_{19} \mathrm{H}_{20} \mathrm{CINO}_{3}$ (345.11): C, 65.99; H, 5.83; N, 4.05. Found: C, 65.66; H, 6.10; N, 4.26. LC-MS: m/z 345 [M] ${ }^{+}$, $347.12\left[\mathrm{M}^{+}+2\right]$,

\section{4-[(3-(4-chlorophenyl)-5,6-dihydro-4H-1,2-oxazin-6-yl)-methyl]-2-methoxyphenol (5i)}

Obtained from 1c $(0.85 \mathrm{~g}, 5 \mathrm{mmol})$, chloramine- $\mathrm{T} .3 \mathrm{H}_{2} \mathrm{O}(1.43 \mathrm{~g}, 5.1 \mathrm{mmol})$ and $4 \mathrm{c}(0.85 \mathrm{~g}, 5.1 \mathrm{mmol})$ as a colorless solid, Yield 54\%; mp 82-83 ${ }^{\circ} \mathrm{C}$; IR $\left(\mathrm{KBr}, \mathrm{cm}^{-1}\right): 1600(\mathrm{C}=\mathrm{C}), 1645(\mathrm{C}=\mathrm{N}), 2990\left(\mathrm{CH}\right.$-aliph.), $3456(\mathrm{HO}) .{ }^{1} \mathrm{H}-\mathrm{NMR}(300 \mathrm{MHz}$, $\left.\mathrm{CDCl}_{3}, \delta / \mathrm{ppm}\right):$ 1.87-2.02 (m, 2H, OCHCH 2$), 2.66-2.74\left(\mathrm{t}, J=7.2 \mathrm{~Hz}, 2 \mathrm{H}, \mathrm{CCH}_{2}\right), 2.78-2.87\left(\mathrm{~m}, 2 \mathrm{H}, \mathrm{PhCH}_{2}\right), 3.75-3.84(\mathrm{~m}$, $1 \mathrm{H}, \mathrm{OCH}), 3.90\left(\mathrm{~s}, 3 \mathrm{H}, \mathrm{CH}_{3}\right), 5.51(\mathrm{~s}, 1 \mathrm{H}, \mathrm{OH}), 6.60-6.70(\mathrm{~m}, 3 \mathrm{H}, \mathrm{Ar}-\mathrm{H}), 7.45(\mathrm{~d}, J=6 \mathrm{~Hz}, 2 \mathrm{H}, \mathrm{Ar}-\mathrm{H}), 7.62(\mathrm{~d}, J=6 \mathrm{~Hz}, 2 \mathrm{H}$, Ar-H). ${ }^{13} \mathrm{C}$ NMR (300 MHz, $\left.\mathrm{CDCl}_{3}\right) ; 15.7,23.9,41.4,58.6,85.8,112.2,117.8,120.9,124.8,128.5,130.1,133.9,136.5$, 147.7, 149.3, 166.9. Anal. Calcd. for $\mathrm{C}_{18} \mathrm{H}_{18} \mathrm{CINO}_{3}$ (331.10): $\mathrm{C}, 65.16 ; \mathrm{H}, 5.47 ; \mathrm{N}, 4.22$. Found: $\mathrm{C}, 65.40 ; \mathrm{H}, 5.30 ; \mathrm{N}, 4.50$. LC-MS: $\mathrm{m} / \mathrm{z} 331[\mathrm{M}]^{+}, 333.12\left[\mathrm{M}^{+}+2\right]$.

\section{3-benzyl-4, 4a, 5,6, 7,8-hexahydro-3H-benzo[c][1,2]oxazine (5j)}

Obtained from 1d $(0.56 \mathrm{~g}, 5 \mathrm{mmol})$, chloramine- $\mathrm{T} .3 \mathrm{H}_{2} \mathrm{O}(1.43 \mathrm{~g}, 5.1 \mathrm{mmol})$ and $4 \mathrm{a}(0.6 \mathrm{~g}, 5.1 \mathrm{mmol})$ as a pale yellow solid, Yield 61\%; mp $89^{\circ} \mathrm{C}$; IR $\left(\mathrm{KBr}, \mathrm{cm}^{-1}\right)$ : $1598(\mathrm{C}=\mathrm{C}), 1644(\mathrm{C}=\mathrm{N}), 2998\left(\mathrm{CH}\right.$-aliph.). ${ }^{1} \mathrm{H}-\mathrm{NMR}(300 \mathrm{MHz}, \mathrm{CDCl}, \mathrm{\delta} / \mathrm{ppm})$ : 1.28-1.36 (m, $1 \mathrm{H}, \mathrm{CCH}), 1.39-1.41\left(\mathrm{t}, \mathrm{J}=7.2 \mathrm{~Hz}, 2 \mathrm{H}, \mathrm{CCH}_{2}\right), 1.49-1.55\left(\mathrm{~m}, 2 \mathrm{H}, \mathrm{CH}_{2}\right), 1.59-1.64\left(\mathrm{~m} .2 \mathrm{H}, \mathrm{CH}_{2}\right), 1.70-1.77(\mathrm{~m}$ $\left.2 \mathrm{H}, \mathrm{CH}_{2}\right), 1.86-2.09\left(\mathrm{~m}, 2 \mathrm{H}, \mathrm{OCHCH}_{2}\right), 2.80-2.87\left(\mathrm{~m}, 2 \mathrm{H}, \mathrm{PhCH}_{2}\right), 3.77-3.88(\mathrm{~m} 1 \mathrm{H}, \mathrm{OCH}), 7.20(\mathrm{t}, \mathrm{J}=6 \mathrm{~Hz}, 2 \mathrm{H}, \mathrm{Ar}-\mathrm{H})$, $7.33(\mathrm{~m}, 3 \mathrm{H}, \mathrm{Ar}-\mathrm{H}) .{ }^{13} \mathrm{C}$ NMR $\left(300 \mathrm{MHz}, \mathrm{CDCl}_{3}\right) ; 21.4,26.6,27.8,32.9,36.9,40.9,41.9,84.6,125.4,128.9,130.9,140.7$, 163,9. Anal. Calcd. for $\mathrm{C}_{15} \mathrm{H}_{19} \mathrm{NO}$ (229.15): C, 78.56; H, 8.35; N, 6.11. Found: C, 78.66; H, 8.20; N, 6.30. LC-MS: m/z 230.26 [M] $]^{+}$.

\section{3-(3,4-dimethoxybenzyl)-4,4a,5,6,7,8-hexahydro-3H-benzo[c][1,2]oxazine (5k)}

Obtained from 1d $(0.56 \mathrm{~g}, 5 \mathrm{mmol})$, chloramine- $\mathrm{T} .3 \mathrm{H}_{2} \mathrm{O}(1.43 \mathrm{~g}, 5.1 \mathrm{mmol})$ and $\mathbf{4 b}(0.9 \mathrm{~g}, 5.1 \mathrm{mmol})$ as a white solid, Yield 69\%; mp 89-91 ${ }^{\circ} \mathrm{C}$; IR ( $\left.\mathrm{KBr}, \mathrm{cm}^{-1}\right): 1600(\mathrm{C}=\mathrm{C}), 1648(\mathrm{C}=\mathrm{N}), 2995\left(\mathrm{CH}\right.$-aliph.). ${ }^{1} \mathrm{H}-\mathrm{NMR}\left(300 \mathrm{MHz}, \mathrm{CDCl}_{3}, \delta / \mathrm{ppm}\right): 1.24-$ $1.32(\mathrm{~m}, 1 \mathrm{H}, \mathrm{CCH}), 1.37-1.40\left(\mathrm{t}, J=7.2 \mathrm{~Hz}, 2 \mathrm{H}, \mathrm{CCH}_{2}\right), 1.47-1.56\left(\mathrm{~m}, 2 \mathrm{H}, \mathrm{CH}_{2}\right), 1.61-1.67\left(\mathrm{~m} .2 \mathrm{H}, \mathrm{CH}_{2}\right), 1.71-1.79(\mathrm{~m}, 2 \mathrm{H}$, $\left.\mathrm{CH}_{2}\right), 1.84-2.01\left(\mathrm{~m}, 2 \mathrm{H}, \mathrm{OCHCH}_{2}\right), 2.78-2.85\left(\mathrm{~m}, 2 \mathrm{H}, \mathrm{PhCH}_{2}\right), 3.72-3.87(\mathrm{~m}, 1 \mathrm{H}, \mathrm{OCH}), 3.85-3.93\left(\mathrm{~m}, 6 \mathrm{H}, \mathrm{OCH}_{3}\right), 6.50-$ 
$6.62(\mathrm{~m}, 3 \mathrm{H}, \mathrm{Ar}-\mathrm{H}) .{ }^{13} \mathrm{C}$ NMR $\left(300 \mathrm{MHz}, \mathrm{CDCl}_{3}\right) ; 21.8,23.3,28.8,31.1,38.8,40.3,42.9,57.2,83.5,114.1,116.5,126.6$, 133.3, 148.2, 149.9, 164.8. Anal. Calcd. for $\mathrm{C}_{17} \mathrm{H}_{23} \mathrm{NO}_{3}$ (289.17): C,70.56; $\mathrm{H}, 8.01 ; \mathrm{N}, 4.84$. Found: $\mathrm{C}, 70.66 ; \mathrm{H}, 7.90 ; \mathrm{N}$, 4.99. LC-MS: $\mathrm{m} / \mathrm{z} 290.29[\mathrm{M}]^{+}$

\section{4-[(4,4a,5,6,7,8-hexahydro-3H-benzo[c][1,2\}oxazine-3-yl)methyl]-2-methoxyphenol (5I)}

Obtained from $1 \mathrm{~d}(0.56 \mathrm{~g}, 5 \mathrm{mmol})$, chloramine-T. $3 \mathrm{H}_{2} \mathrm{O}(1.43 \mathrm{~g}, 5.1 \mathrm{mmol})$ and $4 \mathrm{c}(0.85 \mathrm{~g}, 5.1 \mathrm{mmol})$ as a colorless solid Yield 66\%; mp111-112 ${ }^{\circ} \mathrm{C}$; IR $\left(\mathrm{KBr}, \mathrm{cm}^{-1}\right)$ : $1600(\mathrm{C}=\mathrm{C}), 1645(\mathrm{C}=\mathrm{N}), 2995\left(\mathrm{CH}\right.$-aliph.), $3370(\mathrm{HO}) .{ }^{1} \mathrm{H}-\mathrm{NMR}(300 \mathrm{MHz}$, $\left.\mathrm{CDCl}_{3}, \delta / \mathrm{ppm}\right): 1.23-1.33(\mathrm{~m}, 1 \mathrm{H}, \mathrm{CCH}), 2.72-2.89\left(\mathrm{~m}, 2 \mathrm{H}, \mathrm{PhCH}_{2}\right), 1.38-1.42\left(\mathrm{t}, J=7.2 \mathrm{~Hz}, 2 \mathrm{H}, \mathrm{CCH}_{2}\right), 1.48-1.57(\mathrm{~m}, 2 \mathrm{H}$, $\left.\mathrm{CH}_{2}\right), 1.60-1.65\left(\mathrm{~m} .2 \mathrm{H}, \mathrm{CH}_{2}\right), 1.71-1.78\left(\mathrm{~m}, 2 \mathrm{H}, \mathrm{CH}_{2}\right), 1.82-2.03\left(\mathrm{~m}, 2 \mathrm{H}, \mathrm{OCHCH}_{2}\right), 3.74-3.85(\mathrm{~m}, 1 \mathrm{H}, \mathrm{OCH}), 3.89(\mathrm{~s}, 3 \mathrm{H}$, $\left.\mathrm{OCH}_{3}\right), 5.75(\mathrm{~s}, 1 \mathrm{H}, \mathrm{OH}), 6.65-6.76(\mathrm{~m}, 3 \mathrm{H}, \mathrm{Ar}-\mathrm{H}) .{ }^{13} \mathrm{C} \mathrm{NMR}\left(300 \mathrm{MHz}, \mathrm{CDCl}_{3}\right) ; 22.9,24.9,28.9,31.5,36.6,41.7,42.3$, 58.9, 83.7, 116.3, 118.8, 126.6, 131.1, 145.3, 148.8, 164.9. Anal. Calcd. for $\mathrm{C}_{16} \mathrm{H}_{21} \mathrm{NO}_{3}(275.15): \mathrm{C}, 69.79 ; \mathrm{H}, 7.69 ; \mathrm{N}$, 5.09. Found: C, 69.66; H, 7.80; N, 4.90. LC-MS: m/z $276.27[\mathrm{M}]^{+}$.

\section{3-benzyl-3,4,4a,5,6,7- hexahydrocyclopenta[c][1,2\}oxazine (5m)}

Obtained from 1e $(0.5 \mathrm{~g}, 5 \mathrm{mmol})$, chloramine- $\mathrm{T} .3 \mathrm{H}_{2} \mathrm{O}(1.43 \mathrm{~g}, 5.1 \mathrm{mmol})$ and $4 \mathrm{a}(0.6 \mathrm{~g}, 5.1 \mathrm{mmol})$ as a white solid, Yield 64\%; mp 132-133 ${ }^{\circ} \mathrm{C}$; IR $\left(\mathrm{KBr}, \mathrm{cm}^{-1}\right): 1598(\mathrm{C}=\mathrm{C}), 1648(\mathrm{C}=\mathrm{N}), 2992\left(\mathrm{CH}\right.$-aliph.). ${ }^{1} \mathrm{H}-\mathrm{NMR}\left(300 \mathrm{MHz}, \mathrm{CDCl}_{3}, \delta / \mathrm{ppm}\right): 1.40-$ $1.50\left(\mathrm{~m}, 2 \mathrm{H}, \mathrm{CH}_{2}\right), 1.55-1.60\left(\mathrm{~m}, 2 \mathrm{H}, \mathrm{CH}_{2}\right), 1.66-1.72(\mathrm{~m}, 1 \mathrm{H}, \mathrm{CCH}), 1.85-1.94\left(\mathrm{~m}, 2 \mathrm{H}, \mathrm{OCHCH}_{2}\right), 2.75-2.79(\mathrm{t}, \mathrm{J}=6.2 \mathrm{~Hz}$, $\left.2 \mathrm{H}, \mathrm{CCH}_{2}\right), 2.83-2.89\left(\mathrm{~m}, 2 \mathrm{H}, \mathrm{PhCH}_{2}\right), 3.80-3.87(\mathrm{~m}, 1 \mathrm{H}, \mathrm{OCH}), 7.22(\mathrm{t}, \mathrm{J}=6 \mathrm{~Hz}, 2 \mathrm{H}, \mathrm{Ar}-\mathrm{H}), 7.33(\mathrm{~m}, 3 \mathrm{H}, \mathrm{Ar}-\mathrm{H}) .{ }^{13} \mathrm{C} \mathrm{NMR}$ $\left(300 \mathrm{MHz}_{2} \mathrm{CDCl}_{3}\right) ; 21.8,25.5,29.9,41.8,42.5,44.9,84.9,122.2,126.6,130.2,140.4,166.9$. Anal. Calcd. for $\mathrm{C}_{14} \mathrm{H}_{17} \mathrm{NO}$ (215.13): C, 78.10; H, 7.96; N, 6.51. Found: C, 78.20; H, 7.77; N, 6.88. LC-MS: m/z 216.23 [M] $]^{+}$

\section{3-(3,4-dimethoxybenzyl)-3,4,4a,5,6,7-hexahydrocyclopenta[c][1,2]oxazine (5n)}

Obtained from 1e $(0.5 \mathrm{~g}, 5 \mathrm{mmol})$, chloramine-T. $3 \mathrm{H}_{2} \mathrm{O}(1.43 \mathrm{~g}, 5.1 \mathrm{mmol})$ and $4 \mathrm{~b}(0.9 \mathrm{~g}, 5.1 \mathrm{mmol})$ as a colorless solid Yield 65\%; mp 97-98 ${ }^{\circ} \mathrm{C}$; IR $\left(\mathrm{KBr}, \mathrm{cm}^{-1}\right): 1600(\mathrm{C}=\mathrm{C}), 1649(\mathrm{C}=\mathrm{N}), 2990\left(\mathrm{CH}\right.$-aliph.). ${ }^{1} \mathrm{H}-\mathrm{NMR}\left(300 \mathrm{MHz}, \mathrm{CDCl}_{3}, \delta / \mathrm{ppm}\right)$ : 1.42-1.55 (m, $\left.2 \mathrm{H}, \mathrm{CH}_{2}\right), 1.59-1.64\left(\mathrm{~m}, 2 \mathrm{H}, \mathrm{CCH}_{2}\right), 1.67-1.73(\mathrm{~m}, 1 \mathrm{H}, \mathrm{CCH}), 2.72-2.76\left(\mathrm{t}, \mathrm{J}=6.2 \mathrm{~Hz}, 2 \mathrm{H}, \mathrm{CH}_{2}\right), 1.82-1.91$ $\left(\mathrm{m}, 2 \mathrm{H}, \mathrm{OCHCH}_{2}\right), 2.79-2.85\left(\mathrm{~m}, 2 \mathrm{H}, \mathrm{PhCH}_{2}\right), 3.84-3.87(\mathrm{~m}, 1 \mathrm{H}, \mathrm{OCH}), 3.90-3.93\left(\mathrm{~m}, 6 \mathrm{H}, \mathrm{OCH}_{3}\right), 6.55-6.65(\mathrm{~m}, 3 \mathrm{H}, \mathrm{Ar}-$ $\mathrm{H}) .{ }^{13} \mathrm{C}$ NMR $\left(300 \mathrm{MHz} \mathrm{CDCl}_{3}\right) ; 22.8,26.6,30.3,39.6,41.2,46.6,83.9,127.9,129.9,131.1,135.7,167.9$, 169.4. Anal. Calcd. for $\mathrm{C}_{16} \mathrm{H}_{21} \mathrm{NO}_{3}$ (275.15): C, 69.79; $\mathrm{H}$,7.69; N, 5.09. Found: C, 69.99; H, 7.50; N, 5.40. LC-MS: m/z276.27 [M] .

\section{4-[(3,4,4a,5,6,7- hexahydrocyclopenta[c][1,2]oxazin-3-yl)methyl]-2-methoxyphenol (5o)}

Obtained from 1e $(0.5 \mathrm{~g}, 5 \mathrm{mmol})$, chloramine-T. $3 \mathrm{H}_{2} \mathrm{O}(1.43 \mathrm{~g}, 5.1 \mathrm{mmol})$ and $4 \mathrm{c}(0.86 \mathrm{~g}, 5.1 \mathrm{mmol})$ as a colorless solid,Yield 61\%; mp 112-113 ${ }^{\circ} \mathrm{C}$; IR $\left(\mathrm{KBr}, \mathrm{cm}^{-1}\right)$ : $1600(\mathrm{C}=\mathrm{C}), 1645(\mathrm{C}=\mathrm{N}), 2995$ (CH-aliph.), 3460 (HO). ${ }^{1} \mathrm{H}-\mathrm{NMR}(300$ $\left.\mathrm{MHz}, \mathrm{CDCl}_{3}, \delta / \mathrm{ppm}\right): 1.67-1.76(\mathrm{~m}, 1 \mathrm{H}, \mathrm{CCH}), 2.72-2.77\left(\mathrm{t}, \mathrm{J}=6.2 \mathrm{~Hz}, 2 \mathrm{H}, \mathrm{CH}_{2}\right), 1.53-1.65\left(\mathrm{~m}, 2 \mathrm{H}, \mathrm{CH}_{2}\right), 1.42-1.54(\mathrm{~m}$, $\left.2 \mathrm{H}, \mathrm{CH}_{2}\right), 1.84-1.93\left(\mathrm{~m}, 2 \mathrm{H}, \mathrm{OCHCH}_{2}\right), 2.77-2.88\left(\mathrm{~m}, 2 \mathrm{H}, \mathrm{PhCH}_{2}\right), 3.82-3.87(\mathrm{~m}, 1 \mathrm{H}, \mathrm{OCH}), 3.93\left(\mathrm{~s}, 3 \mathrm{H}, \mathrm{OCH}_{3}\right), 5.45(\mathrm{~s}$,

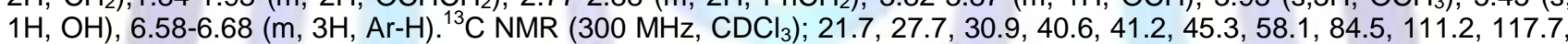
120.9, 133,3, 144.5, 151.2, 168.2. Anal. Calcd. for $\mathrm{C}_{15} \mathrm{H}_{19} \mathrm{NO}_{3}(261.14)$ : $\mathrm{C}, 68.94 ; \mathrm{H}, 7.33 ; \mathrm{N}, 5.36$. Found: $\mathrm{C}, 68.66 ; \mathrm{H}$, 7.60; N, 5.20. LC-MS: m/z $262.27\left[\mathrm{M}^{+}\right.$.

\section{3-benzyl-4,4a,5,6-tetrahydro-3H-Naphtho[1,2-c][1,2]oxazine (3p)}

Obtained from 1f $(0.8 \mathrm{~g}, 5 \mathrm{mmol})$, chloramine-T. $3 \mathrm{H}_{2} \mathrm{O}(1.43 \mathrm{~g}, 5.1 \mathrm{mmol})$ and $4 \mathrm{a}(0.6 \mathrm{~g}, 5.1 \mathrm{mmol})$ as a brown solid, Yield 73\%; mp 140-141 ${ }^{\circ} \mathrm{C}$; IR $\left(\mathrm{KBr}, \mathrm{cm}^{-1}\right): 1600(\mathrm{C}=\mathrm{C}), 1640(\mathrm{C}=\mathrm{N}), 2995$ (CH-aliph.). ${ }^{1} \mathrm{H}-\mathrm{NMR}\left(300 \mathrm{MHz}^{\mathrm{CDCl}} \mathrm{CD}_{3}, \mathrm{\delta} / \mathrm{ppm}\right):$ 1.24-1.35 (m, $1 \mathrm{H}, \mathrm{CCH}), 1.46-1.56\left(\mathrm{t}, J=6.2 \mathrm{~Hz}, 2 \mathrm{H}, \mathrm{CH}_{2}\right), 1.70-1.78\left(\mathrm{~m}, 2 \mathrm{H}, \mathrm{CH}_{2}\right), 1.85-2.02\left(\mathrm{~m}, 2 \mathrm{H}, \mathrm{OCHCH}_{2}\right), 2.77-2.88$ $\left(\mathrm{m}, 2 \mathrm{H}, \mathrm{PhCH}_{2}\right), 3.82-3.87(\mathrm{~m}, 1 \mathrm{H}, \mathrm{OCH}), 7.10(\mathrm{~m}, 3 \mathrm{H}, \mathrm{Ar}-\mathrm{H}), 7.22(\mathrm{t}, J=6 \mathrm{~Hz}, 2 \mathrm{H}, \mathrm{Ar}-\mathrm{H}), 7.35(\mathrm{~m}, 3 \mathrm{H}, \mathrm{Ar}-\mathrm{H}), 7.90(\mathrm{~d}, J=$ $6 \mathrm{~Hz}, 1 \mathrm{H}, \mathrm{Ar}-\mathrm{H}) .{ }^{13} \mathrm{C}$ NMR $\left(300 \mathrm{MHz}, \mathrm{CDCl}_{3}\right) ; 27.2,29.9,32.1,33.9,40.4,80.7,123.9,127.7,128.2,129.1,129.9,131.2$, 131.9, 132.6, 139.1, 144,8, 155,5. Anal. Calcd. for $\mathrm{C}_{19} \mathrm{H}_{19} \mathrm{NO}$ (277.15): $\mathrm{C}, 82.28 ; \mathrm{H}, 6.90 ; \mathrm{N}, 5.05$. Found $\mathrm{C}, 82.56 ; \mathrm{H}$, 6.60; N, 5.20. LC-MS: $\mathrm{m} / \mathrm{z} 278.27[\mathrm{M}]^{+}$.

\section{3-(3,4-dimethoxybenzyl)-4,4a,5,6-tetrahydro-3H-Naphtho[1,2-c][1,2]oxazine (3q)}

Obtained from $1 \mathrm{f}(0.8 \mathrm{~g}, 5 \mathrm{mmol})$, chloramine-T.3 $\mathrm{H}_{2} \mathrm{O}(1.43 \mathrm{~g}, 5.1 \mathrm{mmol})$ and $\mathbf{4 b}(0.9 \mathrm{~g}, 5.1 \mathrm{mmol})$ as a colorless solid,Yield 66\%; mp 131-132 ${ }^{\circ} \mathrm{C}$; IR $\left(\mathrm{KBr}, \mathrm{cm}^{-1}\right): 1600(\mathrm{C}=\mathrm{C}), 1648(\mathrm{C}=\mathrm{N}), 2990\left(\mathrm{CH}\right.$-aliph.). ${ }^{1} \mathrm{H}-\mathrm{NMR}\left(300 \mathrm{MHz} \mathrm{CDCl}_{3}\right.$, $\delta / p p m): 1.26-1.34(\mathrm{~m}, 1 \mathrm{H}, \mathrm{CCH}), 1.45-.155\left(\mathrm{t}, \mathrm{J}=6.2 \mathrm{~Hz}, 2 \mathrm{H}, \mathrm{CH}_{2}\right), 1.72 .1 .81\left(\mathrm{~m}, 2 \mathrm{H}, \mathrm{CH}_{2}\right), 1.84-2.03(\mathrm{~m}, 2 \mathrm{H}, \mathrm{OCHCH})$, 2.77-2.88 (m, $\left.2 \mathrm{H}, \mathrm{PhCH}_{2}\right)$, 3.82-3.87 (m, $\left.1 \mathrm{H}, \mathrm{OCH}\right), 3.85-3.92\left(\mathrm{~m}, 6 \mathrm{H}, \mathrm{OCH}_{3}\right), 6.50-6.60(\mathrm{~m}, 3 \mathrm{H}, \mathrm{Ar}-\mathrm{H}), 7.14(\mathrm{~m}, 2 \mathrm{H}, \mathrm{Ar}-$ $\mathrm{H}), 7.95(\mathrm{~d}, J=6 \mathrm{~Hz}, 1 \mathrm{H}, \mathrm{Ar}-\mathrm{H}) .{ }^{13} \mathrm{C}$ NMR $\left(300 \mathrm{MHz}, \mathrm{CDCl}_{3}\right) ; 28.1,29.9,31.1,33.3,40.9,58.9,79.7,111.9,113.3,123.8$, 124.4, 125.3, 129.4, 129.9, 132.3, 138.8, 146.5, 150.4, 155.8. Anal. Calcd. for $\mathrm{C}_{21} \mathrm{H}_{23} \mathrm{NO}_{3}(337.17): \mathrm{C}, 74.75 ; \mathrm{H}, 6.87 ; \mathrm{N}$, 4.15; Found: C, 74.66; H, 6.90; N, 4.10. LC-MS: $\mathrm{m} / \mathrm{z} 338.28[\mathrm{M}]^{+}$.

\section{2-methoxy-4-[(4,4a,5,6-tetrahydro-3H-Naptho [1,2-c][1,2] oxazinyl)-methyl] phenol (3r)}

Obtained from 1f $(0.8 \mathrm{~g}, 5 \mathrm{mmol})$, chloramine- $\mathrm{T} .3 \mathrm{H}_{2} \mathrm{O}(1.43 \mathrm{~g}, 5.1 \mathrm{mmol})$ and $4 \mathrm{c}(0.85 \mathrm{~g}, 5.1 \mathrm{mmol})$ as a colorless solid,Yield 69\%; mp 125-127 ${ }^{\circ} \mathrm{C}$; IR $\left(\mathrm{KBr}, \mathrm{cm}^{-1}\right)$ : $1600(\mathrm{C}=\mathrm{C}), 1648(\mathrm{C}=\mathrm{N}), 2990$ (CH-aliph.), $3465(\mathrm{HO}) .{ }^{1} \mathrm{H}-\mathrm{NMR}(300$ $\left.\mathrm{MHz}_{\mathrm{CDCl}}, \delta / \mathrm{ppm}\right): 1.21-1.34(\mathrm{~m}, 1 \mathrm{H}, \mathrm{CCH}), 1.45-1.56\left(\mathrm{t}, \mathrm{J}=6.2 \mathrm{~Hz}, 2 \mathrm{H}, \mathrm{CH}_{2}\right), 1.70-1.82\left(\mathrm{~m}, 2 \mathrm{H}, \mathrm{CH}_{2}\right), 1.84-1.99(\mathrm{~m}$, $\left.2 \mathrm{H}, \mathrm{OCHCH}_{2}\right), 2.77-2.88\left(\mathrm{~m}, 2 \mathrm{H}, \mathrm{PhCH}_{2}\right), 3.82-3.87(\mathrm{~m}, 1 \mathrm{H}, \mathrm{OCH}), 3.86\left(\mathrm{~s}, 3 \mathrm{H}, \mathrm{OCH}_{3}\right), 5.55(\mathrm{~s}, 1 \mathrm{H}, \mathrm{OH}), 6.60-6.71(\mathrm{~m}$, $3 \mathrm{H}, \mathrm{Ar}-\mathrm{H}), 7.18-7.80(\mathrm{~m}, 3 \mathrm{H}, \mathrm{Ar}-\mathrm{H}), 7.95(\mathrm{~d}, \mathrm{~J}=6 \mathrm{~Hz}, 1 \mathrm{H}, \mathrm{Ar}-\mathrm{H}) .{ }^{13} \mathrm{C}$ NMR $\left(300 \mathrm{MHz}, \mathrm{CDCl}_{3}\right) ; 27.7,30.1,31.2,33.2,41.4$, 57.7, 78.9, 112.9, 116.8, 121.2, 123.3, 126.7, 128,2, 129.9, 131.1, 132.2, 140.5, 146.4, 149.9, 156. Anal. Calcd. for $\mathrm{C}_{20} \mathrm{H}_{21} \mathrm{NO}_{3}$ (323.15): C, 74.28; $\mathrm{H}, 6.55 ; \mathrm{N}, 4.36$. Found: C, 74.66; H, 6.40; N, 4.50. LC-MS: m/z 324.26 [M] ${ }^{+}$. 


\section{RESULTS AND DISCUSSION}

The compounds $\mathbf{1}(\mathrm{a}-\mathrm{f})$ were synthesized by the reaction of ketones with hydroxylamine hydrochloride in the presence of sodium acetate. Chloramine-T was used as an effective reagent for the generation of a-nitrosolefins from the corresponding ketoximes 1(a-f) [31], which subsequently undergo hetero Diels-Alder reaction with an olefin 4(a-c) to give 5,6-dihydro-4H-1,2-Oxazine derivatives 5(a-r) (Scheme 1) [32].<smiles>[R]C(=N)C(=O)O</smiles>

$1(a-f)$<smiles>[R]CC([R])(CC)N=O</smiles><smiles>[R]/C=C(/[R])N=O</smiles>

3

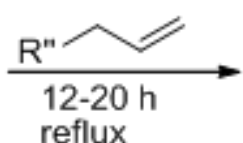

$4(\mathrm{a}-\mathrm{c})$<smiles>[R]CC1CC([R])C([R])=NO1</smiles>

$5(a-r)$ a $\mathrm{R}^{\prime \prime}=\mathrm{Ph}$

b R" =<smiles>COc1ccc(C)cc1OC</smiles>

The regioselectivity can be interpreted in terms of interaction between the FMO of diene and dienophile. Usually, for dipolarophiles with electron-attracting groups, the dipolar-HOMO and dienophile-LUMO interaction is dominant. According to the principle of maximum overlap, the preferred isomers of each interaction can be predicted by union of two sites of the reactants having the largest coefficient value. Most dienophiles undergo reaction to give 6-substituted 1,2-oxazines with high regioselectivity[33]. The identities of compounds (5a-r) were established by their ${ }^{1} \mathrm{H} N M R$, ${ }^{13} \mathrm{C}$ NMR, IR, elemental analysis and MS. Taking $5 \mathrm{a}$ as an example, the ${ }^{1} \mathrm{H}$ NMR spectrum of 5 a displays triplet at 2.60-2.74 and multiplets at 1.82-2.04 and 3.75-3.82 ppm due to $\mathrm{CCH}_{2}, \mathrm{OCHCH}_{2}$ and $\mathrm{OCH}$ in the position 4, 5 and 6 in the oxazine ring respectively. While the $\mathrm{CH}_{2}-\mathrm{Ph}$ appears as multiplet at $2.78-2.86 \mathrm{ppm}$. Further the ten protons of two phenyl groups appeared at $\delta 7.20$ 7.73 as a multiplet, The IR spectra of 1,2-oxazine 5a showed absorption at $1640 \mathrm{~cm}^{-1}$ owing to the ring nitrogen( $\mathrm{C}=\mathrm{N}$ ) and $1600 \mathrm{~cm}^{-1}$ due to aromatic ring. In the ${ }^{13} \mathrm{C}$ NMR spectra all oxazines gave consistent signals for the newly formed ring carbons. For instance the signals in the region due to $\mathrm{C}_{6}$ appears in the region $\delta 86.90 \mathrm{ppm}$ while $\mathrm{C}_{5}, \mathrm{C}_{4}$ and $\mathrm{C}_{3}$ appears in the region 17.2, 24.2 and 165.30. The mass spectrum of $\mathbf{5 a}$ showed the molecular ion peak at $\mathrm{m} / \mathrm{z} 252.26$. The formation of the products was further supported by elemental analysis.

\section{CONCLUSIONS}

In conclusion a new series of 5,6-dihydro-4H-1,2-oxazine derivatives were synthesized from ketooximes by generation of a nitrosolefins and reacting them with derivatives of allylbenzene. We have also prepared bicyclic oxazines from cyclopentanone oxime, cyclohexanone oxime and 3,4-dihydronaphthalen-1(2H)-one oxime.

\section{ACKNOWLEDGMENTS}

Authors wish to thank the University of Mysore, India who provided all the facilities that helped in completing this research work.

\section{REFERENCES}

[1] T.Petros and G. Soungas, Heterocycles, 2002, 57, 1149-1178.

[2] T.Petros, and G. Soungas, Heterocycles, 2002, 57, 915-953.

[3] M.Naruse, S.Aoyagi and C.Kibayashi, Tetrahedron Lett. 1994, 35, 595-598. 
[4] T.L.Gilchrist, Chem. Soc. Rev., 1983,12, 53-73.

[5] G.W. Kirby, Chem. Soc. Rev. 1997, 6, 1-24.

[6] A. Defoin, H.Sarazin, C. Strehler and J.Stretch, Tetrahedron Lett., 1994, 35, 5653-5656

[7] R. Henning, U.Learch and H.Urbach, Synthesis, 1989, 265-268.

[8] P.Bach, and M. Bols, Tetrahedron Lett.,1999, 40, 3461-3464.

[9] M.Miyashita, B.Z.E.Awen and A.Yoshikoshi, Tetrahedron, 1990, 46, 7569-7586.

[10] K.M.Patel and T.M. Stevenson, Herbicidal oxazine ethers. U.S. Patent 5510318 April 23, 1996.

[11] R.Fischer, T.Bretschneider, F.Lieb, T.Schenke, A.Ullmann-Koppold, M.Wilhelm Drewes, D.Feuch, J.Konze, P. Losel, O.

Malsam, K-H.Kuck, A.Wachendorff- Neumann, A.Angermann, C.Arnold, T.Auler, H.Hempel, K.Hills and E.Sanwald, Phenyl-substituted [1,2]-oxazine-3,5-dione and dihydropyrone derivatives, U.S.Patent 0026943 Jan. 31, 2008.

[12] R.Zimmer, M.Colias, M. Roth and H.U.Reissig, Liebigs Ann. Chem., 1992,709-714.

[13] W.Oppolzer, K.Battig and K.Hudlicky, Tetrahedron, 1981, 37, 4359-4364.

[14] S.Nakanishi, Y.Shirai, K.Takahashi and Y.Otsuji, Chem. Lett., 1981, 10, 869-872.

[15] J.Angemann, K.Homann and H.U.Reissig, R. Zimmer, Synlett. 1995, 10, 1014-016.

[16] R.Faragher and T.L.Gilchrist, J. Chem. Soc. Perkin Trans 1, 1979, 258-262.

[17] T.L.Gilchrist, D.A.Lingham and T.G.Roberts, J. Chem. Soc. Chem. Commun.,1979, 1089-1090.

[18] E.D.David, L.G.Thomas and G.R.Tony, J. Chem. Soc., Perkin Trans. 1, 1983,1275-1281.

[19] T.Shigeo, M.Hideshi and H. Makoto, T. J. Chem. Soc., Perkin Trans. 1, 1991, 3153-3157.

[20] K.Mori and J.Wu, Liebigs Ann. Chem. 1991, 213-217.

[21] (a) W.R.Bowman, R.V.Davies, A.M.Z.Slawin, G.S.Sohal, R.B.Titman and D.J.Wilkins, J. Chem. Soc., Perkin Trans 1 ,1997, 155-161. (b)Tiecco, M.Testaferri, L.Tingoli, M.Bagnoli and L.F.Marini, J. Chem. Soc.,Perkin Trans. 1, 1993,1989, 1993.

[22] R. Bishop, S.C.Hawkins, T.A.O.Quibuyen and P.R.Brooks, Tetrahedron Lett.1988, 29, 6805-6808.

[23] D.Armest, M.A.Austin, O.J.Griffiths, W.M.Horspool and M.Carpintero, J.Chem. Soc., Chem. Commun., 1996, 27152716.

[24] a) A.Kaiser, K.K.Mayer, A.Sellmer and W.Wiegrebe, Monatsh. Chem. 2003, 3, 343.; (b) G.J.Ellames, C. T.Hewkin,; R F. W. Jackson,; D. I.Smith,; S. P.Standen, Tetrahedron Lett., 1989, 30, 3471-3472.

[25] H.Saiki and T.Mukai, Chem. Let .,1981, 1561-1564.

[26] D.Lathbury and T.Gallagher, J. Chem. Soc., Chem. Commun.,1986, 114-115.

[27] V.Castin and C.N.Rentzea, Angew Chem., 1980, 92, 195-197.

[28] F.Boberg, M.Ruhr, K.H.Garburg and A.Garming, J. Heterocycl. Chem., 1986, 23, 759-766.

[29] T.T.Dang, U.Albrecht, K.Gerwien, M.Siebert, P.Langer, J. Org. Chem., 2006, 71, 2293-2301.

[30] S.L.Gaonker and K.M.L.Rai. J. Heterocylic Chem., 2006, 42, 877-881.

[31] S.F.Brian, J.H.Antony, W.G.S.Peter and R.T.Austin, Vogel's Textbook of Organic Chemistry, 5th ed.; Dorling Kindersley (India) Pvt. Ltd.,(2006).[32] M.K.Manjula, K.M.L.Rai, S. L.Gaonkar, K.A.Raveesha and S.Satish Eur. J. Med. Chem., 2009, 44, 280-288.

[33] K.M.Lokanatha. Rai, Heterocycles via oximes cycloadditions, Ed.; Springer- Verlag Berlin Heidelberg, (2008), Vol.13, pp 10. 


\section{Author' biography with Photo}

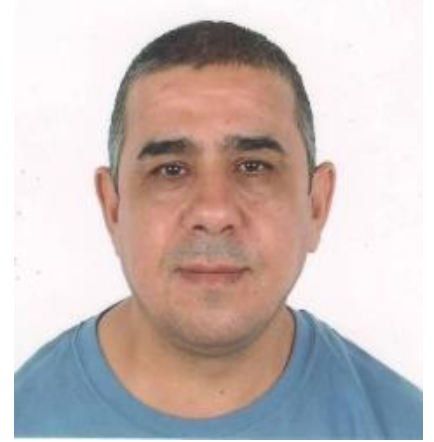

Author's name is Raad Kasim Yhya. He obtained his bachelors and masters in science in chemistry at University of Mosul, Iraq. He has worked as a lecturer at College of Science and Arts at University of Al- marqab, Libya for 13 years from 1997 to 2010 . He is an Iraqi national who is doing his Research in DOS in Chemistry, University of Mysore, Mysore under the guidance of Prof. K. M. Lokanatha Rai. His work mainly deals with Synthetic organic chemistry, Synthesis of heterocycles and cycloaddition reactions. 